\title{
Physical Kinetics, Relativism and Nonlocal Physics
}

\author{
Boris V. Alexeev \\ MIREA, Russian State Technological University, Moscow, Russia \\ Email: Boris.Vlad.Alexeev@gmail.com
}

How to cite this paper: Alexeev, B.V. (2022) Physical Kinetics, Relativism and Nonlocal Physics. Journal of Applied Mathematics and Physics, 10, 68-90. https://doi.org/10.4236/jamp.2022.101007

Received: December 11, 2021

Accepted: January 15, 2022

Published: January 18, 2022

Copyright $(\odot) 2022$ by author(s) and Scientific Research Publishing Inc. This work is licensed under the Creative Commons Attribution International License (CC BY 4.0).

http://creativecommons.org/licenses/by/4.0/

\begin{abstract}
The old classical problems of theoretical physics are revisited from the point of view of nonlocal physics. Nonlocal physics leads to very complicated mathematical apparatus. Here, we explain the main principles of nonlocal physics using transparent considerations and animations.
\end{abstract}

\section{Keywords}

Nonlocal Physics, Transport Processes in the Dissipative Statistical Physical Systems, Relativistic Theories, Michelson-Morley Experiment, The Uncertainty Heisenberg Principle

\section{Introduction: Preliminary Remarks}

By the end of the twentieth century, it was found that $96 \%$ of matter and energy was in the Universe of unknown origin. The terminology "dark matter" and "dark" energy was introduced in the scientific language. But even this would not be such a depressing fact, if it were not for the belief that this dark matter and dark energy are not diagnosed, and only the indirect effects on space objects can judge the existence of dark matter and dark energy. We even had to face the opinion of religious-minded people who claimed that four percent is all that the Creator left for the study to man. In practice, science has faced the most serious challenge since the fundamental monograph of I. Newton "Mathematical principles of natural philosophy" was published in 1687 . On the Internet you can find a reaction to the current situation, bordering on despair.

We should underline that it is not the crisis of theoretical physics. It is the crisis of the local statistical theory of the dissipative processes. I initially assumed that the nonlocal physics developed by me (see for example [1] [2] [3] [4]) would lead to very significant changes in the description of natural phenomena. 
That is to say, $4 \%$ of new information compared to $96 \%$ of the classical theory, but not Vice Versa! What effect as a result! It is impossible to believe that more than three hundred years after Newton, world science came to such a disappointing result. Then the question arises-where is the error? The answer to this question is given by nonlocal physics. In other words, the origin of all difficulties lies in the total oversimplification, which follows from the fundamental shortcomings of the local statistical theory of transport processes.

There is another sad circumstance. In terms of time, the crisis in theoretical physics coincided in my country with an obvious drop in the interest of young people in the exact sciences in general and in physics in particular. Therefore, all my main works are published in English. However, as it turned out, not everything is so bad. There is an extremely useful Quora website. Quora is a social question-and-answer website based in Mountain View, California, United States. The website was made available to the public on June 21, 2010. Users can collaborate by editing questions and commenting on answers that have been submitted by other users. In 2020, the website was visited by 300 million users a month. Quora is aimed not only at the audience of young researchers starting their way in science, but also well-established scientists trying to restore order in the "classical" principles of theoretical physics. Smart and well-founded questions are being asked. Example-Jess H. Brewer (Professor of Physics since 1977) asks:

What are the units of the wave function in Quantum Mechanics? Her comments:

For a 1-dimensional position space wave function $\psi(x)$, the normalization condition would be

$$
\int \psi^{*}(x) \psi(x) \mathrm{d} x=1,
$$

so $\psi^{*}(x) \psi(x)$ has units of inverse distance and $\psi(x)$ has units of square root of inverse distance, e.g. $\mathrm{m}^{-1 / 2}$ (Probability is a pure number; it has no units). For a 3-dimensional position space wave function $\psi(\mathbf{r})$, the normalization condition would be

$$
\int \psi^{*}(\mathbf{r}) \psi(\mathbf{r}) \mathrm{d} \mathbf{r}=1,
$$

so $\psi^{*}(\mathbf{r}) \psi(\mathbf{r})$ has units of inverse volume and $\psi(\mathbf{r})$ has units of square root of inverse volume, e.g. $\mathrm{m}^{-3 / 2}$.

It is an absolutely reasonable question. But this question has no answer in the frame of local physics. The answer sounds as follows:

The single electron can be "smeared" into hydrodynamics (Madelung). From position of nonlocal physics wave function $\psi(\mathbf{r})$ is only a mathematical function (between many others) defining by corresponding relations. The attempt to find any physical sense for wave function has no reason. In the Madelung's interpretation wave function has the dimension $\sqrt{\rho}$ : "square root from the density".

Intermediate conclusions:

1) We need rewriting the teaching physics programme on the basement of 
nonlocal physics.

2) Nonlocal physics developed from the first principles of physics (has no phenomenological form) and leads to the complicated mathematical apparatus.

3) I'm aware that physics is the science of common sense and the main principles of nonlocal physics could be explained without the complicated formulae using animations (as maximum).

4) As a result in all my monographs and papers in introductions I explained the main principles of nonlocal physics using the simplest explanations on the qualitative level (see [1] [2] [3] [4]).

5) Here I follow my explanations on qualitative level for the better understanding of non local theory. In the definite sense the following can be considered as a continuation the article [5].

I repeat-the origin of difficulties in theoretical physics consists in the total Oversimplification following from the principles of local physics and reflects the general shortcomings of the local kinetic transport theory and reflects the general shortcomings of the local kinetic transport theory based on the Boltzmann kinetic theory.

The article also briefly describes the (often tragic) history of the study of complex dissipative systems. As you can see, the attempt to describe the evolution of the universe goes back to St. John, Newton, Kelvin, Boltzmann, Lorentz and Poincaré.

\section{Boltzmann Physical Kinetics, Newton and Apocalypse}

It is well known that by the end of his life Newton was engaged in the interpretation of the Bible, which caused confusion among contemporaries and subsequent biographers of perhaps the greatest genius in the history of mankind. A genius of such magnitude as Newton does not need any excuses, but let's try to imagine the logic of his behavior. By the end of his life, he realized that he had created a scientific picture of the world; equations were found that describe the mechanical motion of the particles that make up the universe, including taking into account gravity. This model also included light effects involving light particles -photons. It would seem that a brilliant opportunity has appeared to deal with the evolution of complex systems and, at the same time, complete hopelessness, if we consider the evolution of the universe.

There are too many equations without initial conditions. Well, if the calculations are not real, then maybe it is possible to understand at the level of insight? Indeed, there are, for example, human counters who perform gigantic calculations, but in general do not even understand how they do it. It's just that in a head, say, when multiplying multi-digit numbers, a series of numbers is built up, which is perceived by the counter as the result of calculations.

Where can one find an answer at the level of insight to the question of the evolution of the universe? Only in the Bible, and above all in the Apocalypse of St. John. This is what Newton did. The results of his interpretations were pub- 
lished only in 1733, that is, 6 years after his death (Observation upon the Prophecies of Daniel and the Apocalypse of St. John, London, 1733). It is an indicative fact that Newton did not dare to publish his reflections during his lifetime. Surely he would have met criticism from two sides-colleagues of natural scientists and theologians who have their own ideas about the Apocalypse that have developed over $\sim 17$ centuries.

This criticism of Newton for his latest work has become a common place in encyclopedias not only of the Marxist kind. For example, in Meyer's lexicon of 1897 (5th edition, Leipzig and Vienna) we read: In his "Reflections on the sermons of Daniel and the Apocalypse of St. John" immersed his clear spirit in mystical visions: "in general, religious reasoning in the later years of Newton's life became his main occupation".

The Apocalypse, of course, is one of the most mysterious and obscure books of the New Testament. According to legend, the apocalyptic visions of the "Revelation of the Apostle John", as an epiphany, descended to John on the island of Patmos in the year 95 of the Birth of Christ.

It is a small island (area $36.9 \mathrm{~km}^{2}$ ) located in the Aegean Sea in the Sporades Island group. The island is completely treeless, hilly, with the highest point at $293 \mathrm{~m}$. Heavy, drying climate, strong winds. At the same time, the island was not a remote, God-forsaken place. The fact is that the rules of coastal navigation of antiquity provided for the need for an anchorage at the end of each day. Patmos just had a good harbor after a one-day crossing from Ephesus on the way to Rome. Let us recall that Ephesus, founded in the second half of the second millennium BC on the western coast of Asia Minor, came under Roman rule in 133 $\mathrm{BC}$ and in the first century $\mathrm{AD}$ became one of the main cities of the province of Asia. A large construction was carried out in the city, which turned it into a large trade and craft center.

According to Victor Guérin, in Hellenic times, the urban population on Patmos numbered 12 - 13 thousand inhabitants. In addition, there were several villages on the island, the population of which reached 4 thousand people. The island's population was gradually declining due to lack of water and poor soil. The port of Patmos has lost its importance, as well as the port of Ephesus, which has become very shallow. At the end of the century before last, there were already less than 400 inhabitants on the island. Orestes built a temple here in honor of Artemis of Scythia. It is assumed that this temple was located on the site of the now existing monastery, built in the 11th century by St. Christodule.

According to legend, at the end of the reign of the Roman emperor Titus Flavius Domitian, new persecutions of Christians began, although perhaps not as cruel as in the time of Nero, when torches were arranged from living Christians to illuminate night races. In 95, John the Theologian was sent into exile on the island of Patmos. There are other versions of the reason for his stay on the island [6]. In September 96, Domitian was assassinated, the Roman Senate revoked his decrees, and the new emperor Nerva returned the exiles. It is believed that John returned to Ephesus in early 97, having stayed on Patmos for about 18 months. 
Now a tourist or a pilgrim will be shown a stone baptistery font, in which John baptized converts. You will be shown the "Holy Cave of Revelation", where John wrote the Apocalypse. Divine services are held in the cave temple, here John slept on the stone floor: a small niche, now decorated with a gilded crown, served as his headboard. Here a voice came from a crack in the rock, commanding him to write down the "Revelation".

The "Revelation" begins with the epistles to the seven churches, after which the vision of the end of the world is presented. The enchanting pictures of the end of the world, painted by the Apostle at the highest artistic level, shocked the imagination, but also baffled so much that attempts were repeatedly made to remove the "Apocalypse from John" from the list of canonical books. Even after the reconciliation of Church and State in the 4th century, attempts are known to declare the "Revelation" an apocryphal book. The origin of these actions is explained, firstly, by possible historical parallels at the Nero-Antichrist level and, secondly, by a strangely inconsistent text, according to commentators. Already Dionysius of Alexandria in the third century writes about the Apocalypse "in a tone of great confusion and admits that he does not understand anything about it" [6]. It is unlikely that historical parallels, imaginary or real, play a primary role in the Apocalypse. This does not fit into the psychological picture of what is happening. Why would an "old Jewish fanatic" (in the words of Ernest Renan), in his declining years on an island lost in the sea, engage in allegories. Why it was impossible to call Nero by name, especially since he committed suicide 27 years before the visions of the Apostle John.

Thus, there is a feeling that the book paints real pictures of the end of the world, as they were presented to John. Let's consider this an epiphany. To paraphrase Renan, we can say: "This is a rough pencil sketch of a child who draws an unusual tool for him, a picture of a city that he has never seen before." Apocalyptic scenes should be treated as a representation of reality at a level accessible to Priest John.

If it was just that, it would be half the trouble. Commentators and, in particular, Renan are annoyed by the inconsistency of the Apocalypse. "Instead of the final denouement that was to follow the opening of the seventh seal, there is silence in heaven, as if for half an hour, as a sign that the first act of the mystery has ended and now the next act must begin."

The same is seen in the Song of Songs. The five acts of this little drama do not go on continuously one after the other. Each is followed by a new beginning of the action, which ends there. "In general, Jewish literature does not know the law of unity of action at all” ([6], p. 295).

Let's now try to deal with this lack of "unity of action" from the standpoint of the nonlocal physics. The reader, far from religion, can consider all the following exposition as science fiction.

Let's turn to Figure 1, which shows the evolution of the $H$-function as it approaches global thermodynamic equilibrium. Roughly speaking, we can consider 


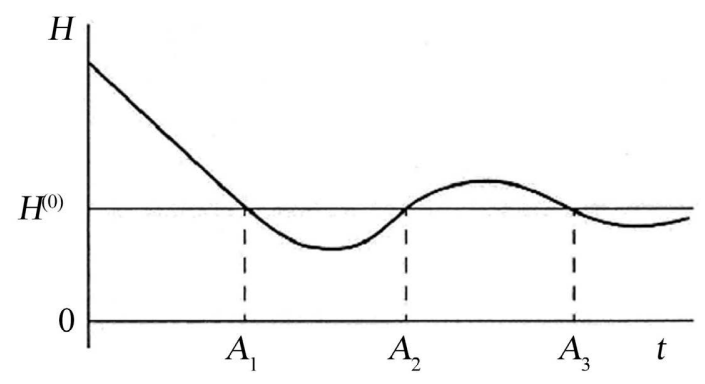

Figure 1. Evolution $H$-function in time when approximated by the predicted future. $H^{(0)}$-equilibrium value of $H$-function.

the $H$-function as the entropy of a system with the opposite sign. At the "apocalyptic point", the $H$-function goes beyond the global thermodynamic equilibrium, which corresponds to the departure of entropy from the equilibrium value.

What preceded the removal of the last seventh seal by the slain Lamb (Jesus Christ)? Terrible disasters have struck the Earth-wars, famine, disease. The moon turned bloody, and the stars of heaven fell to the ground. "The day of great wrath has come and who can survive it?" The heavens split and rolled up like a scroll and all the mountains and islands moved from their places.

But the first apocalyptic point has been passed and the world has not perished, but has turned into some kind of strange looking glass in which the Antichrist reigns, Satan in one of his most formidable incarnations. The power of the Beast comes, and people begin to worship him.

The system is moving further away from the state of global thermodynamic equilibrium. And the second Beast comes out of the earth, which acts with all the power of the first Beast and works miracles "to the point that fire descends from heaven to earth in front of people."

The system completes its evolution after the first apocalyptic point and moves to the second apocalyptic point $A_{2}$. The second transition through the state of global thermodynamic equilibrium is realized. "The harvest of the earth is ripe, the Son of Man has cast his sickle on the earth, and the earth has been reaped" (Renan, [6]). "The beast and the false prophet were thrown alive into a boiling lake of fiery brimstone" (Revelation).

The world is moving away from the state again, but the fluctuation is small and the world is only a pale reflection of its former greatness. "This is the first resurrection from the dead. The rest of the dead did not come back to life." Those who were reborn reigned with Christ for a thousand years.

Evolution brings us to the third apocalyptic point $A_{3}$ and again the system goes under the level $H^{(0)}$. "Then, at the end of a thousand years, Satan will come out of prison and go to deceive the nations scattered on the Earth. But fire descended from heaven and devoured the satanic army. And then Satan, who had deceived these people, was thrown into a lake of boiling sulfur, where the Beast and the False Prophet were. And they will be tormented day and night forever 
and ever."

The providence of the fourth apocalyptic point, in fact, ends the "Revelation". So, John proposed the first model of evolution with damped oscillatory relaxation.

It remains only to note that the model of the evolution of the universe with decaying vibration relaxation is a consequence of the statistical-mechanical picture of the world, which does not exclude other cosmological theories at all.

Surprisingly, far from trivial physical ideas intersect with church traditions. I have already written about the oscillatory approximation of thermodynamic systems to the state of thermodynamic equilibrium in connection with the Apocalypse. But here's another striking idea of the trinity.

Trinity (other-Greek. $\tau \rho$ lás, Lat. Trinitas) is a theological term reflecting the Christian teaching about the three Persons of the essentially one God. For the first time the term "Trinity" is attested by Theophilus of Antioch (II century). It was at the Council of Nicaea that the Creed was approved-a formula that briefly conveys Christian dogmas. There was also approved the dogma of the Trinity: the trinity of God the Father, God the Son and God the Holy Spirit.

This Creed, which fixed the dogma of the Most Holy Trinity, occupies a central place in the liturgical practice of many Christian churches and is the basis of Christian doctrine. According to him:

1) God the Father is not born of anyone and does not come from anyone.

2) God the Son is eternally born of God the Father.

3) God the Holy Spirit eternally comes from God the Father.

All three Hypostases of the Trinity exist in complete unity, which creates the world, cares for it and sanctifies it.

In physics, respectively, we have:

1) Physical vacuum;

2) Matter;

3) All kinds of fields.

\section{Boltzmann's Mistake}

In fact, the mentioned crisis in theoretical physics has evolved over the years. The first heavy blow in this way the unresolved physical problems was the suicide of the great physicist L. Boltzmann; according to the belief of many connected with unsolved problems and criticism of Boltzmann kinetic theory, the first of the following list of unsolved problems of fundamental physics (local physical kinetics of dissipative processes):

1) Kinetic theory of entropy and the problem of "primary" fluctuation.

2) A strict theory of turbulence.

3) Quantum non-relativistic and relativistic hydrodynamics, the theory of charge separation in atomic structures and nuclei. High temperature superconductivity.

4) The theory of ball lightning. 
5) The theory of dark matter.

6) The theory of dark energy. Hubble expansion of the Universe.

7) The destiny of antimatter after the Big Bang.

8) Unified theory of dissipative structures, from atomic structure to cosmology.

These problems cannot be solved within the framework of local physics, but find their natural solution as a special case of solving the problem 8 -the creation of a unified theory of dissipative structures from atomic structures to cosmology.

Let us discuss the situation in kinetic description of the dissipative physical systems in more details.

In physical kinetics, transfer processes in open dissipative systems are considered. Therefore, the kinetic statistical description is inevitably associated with the diagnosis of the system. Let's ask ourselves the question-what is the temperature at some point of the physical system? The question is far from trivial. What is temperature from the point of view of kinetic theory? It is a measure of the average kinetic energy in a physically infinitesimal volume. But what is a physically infinitesimal volume (PhIV)? This volume is large enough to talk about the possibility of reasonable averaging of statistical parameters; but it is also small enough compared to the volume of the entire system to preserve the locality of measurements.

Such an element of diagnostics in the theoretical description in physical kinetics is the concept of a physically infinitesimal volume (PhIV). The connection of the theoretical description with the diagnostics of the system is well known in physics. It is enough to recall the role of the test charge in electrostatics or the test circuit in the physics of magnetic phenomena. The traditional definition of PhIV contains the statement that PhIV $V_{M}$ contains a sufficient number of particles to introduce a statistical description, but at the same time, $\mathrm{PhIV}$ is much smaller than the volume of the physical system under consideration, which provides a local approach to the study of transfer processes. That is why in classical hydrodynamics it is assumed that first a local thermodynamic equilibrium (LTE) is established within the PhIV, and only then comes the transition to global thermodynamic equilibrium, if it is at all possible for the system under study. Thus, it is implicitly assumed that the role of boundary particles in an open thermodynamic system, which is the PhIV, is negligible.

It is often necessary to face the conviction that possible corrections related to boundary effects of the order of the particle diameter, and clarification of the PhIV concept, for example, in the theory of rarefied gases, cannot lead to effects of practical importance. We show that such an interpretation of the classical definition of PhIV is erroneous.

Let us consider this interpretation in more detail, raising the question, as they sometimes say in physical kinetics, "about the size of a point". Let a particle of finite diameter be characterized by the same position $\mathbf{r}$ at the moment of time 
$t$ of its center of mass moving with velocity $\mathbf{v}$. We consider two adjacent physically infinitesimal volumes $V_{\mu, 1}$ and $V_{M, 2}$ in a none-equilibrium system. The volume corresponds to a single-particle distribution function (DF) $f_{m, 1}\left(\mathbf{r}_{1}, \mathbf{v}, t\right)$, and the volume $V_{M, 2}$, respectively, $f_{M, 2}$. In the first approximation, we can assume that $f_{M, 1}$ it does not change within the limits $V_{M, 1}$, just as $f_{M, 2}$ it does not change within the neighboring volume $V_{M, 2}$. It is this assumption that is implicit in Boltzmann's theory.

However, the assumption is too crude. Indeed, a particle at the boundary of two volumes, which experienced the last collision in $V_{s, 1}$ and heading to the side $V_{M, 2}$, introduces information about the DF $f_{M, 1}$ into the neighboring volume $V_{M, 2}$. Similarly, a particle at the boundary of two volumes, which experienced the last collision in $V_{M, 2}$ and heading to the side $V_{M, 1}$, introduces information about the DF $f_{M, 2}$ into the neighboring volume $V_{M, 1}$.

Even point particles (which started moving after the last collision near the transparent boundary between the mentioned volumes) can change the distribution function in the neighboring volume.

Relaxation by translational degrees of freedom of the dynamic characteristics of a particle in a neighboring volume requires several collisions, even in the simplest case of relaxation of particles of the same masses.

In particular, the fact that the center of mass of some particle located near the boundary $\mathrm{PhIV}_{1}$ is in $\mathrm{PhIV}_{1}$ does not mean that the entire particle is in this volume. Then a situation is possible when at some point in time $t$ the particle is located at the interface of two volumes. In this case, an advance effect is possible (say for $V_{u, 2}$ ) when the center of mass of the particle heading to the neighboring volume $V_{\mu, 2}$ is still in $V_{\mu, 1}$. But there is also a lag effect, when the center of mass of a particle heading to a neighboring volume (say $V_{M, 2}$ ) is already in $V_{M, 2}$, but part of the particle still belongs to $V_{M, 1}$. In the "surface" layer of neighboring PhIV's there will always be particles of finite diameter belonging to both the first and second physically small volumes (Figure 2 ).

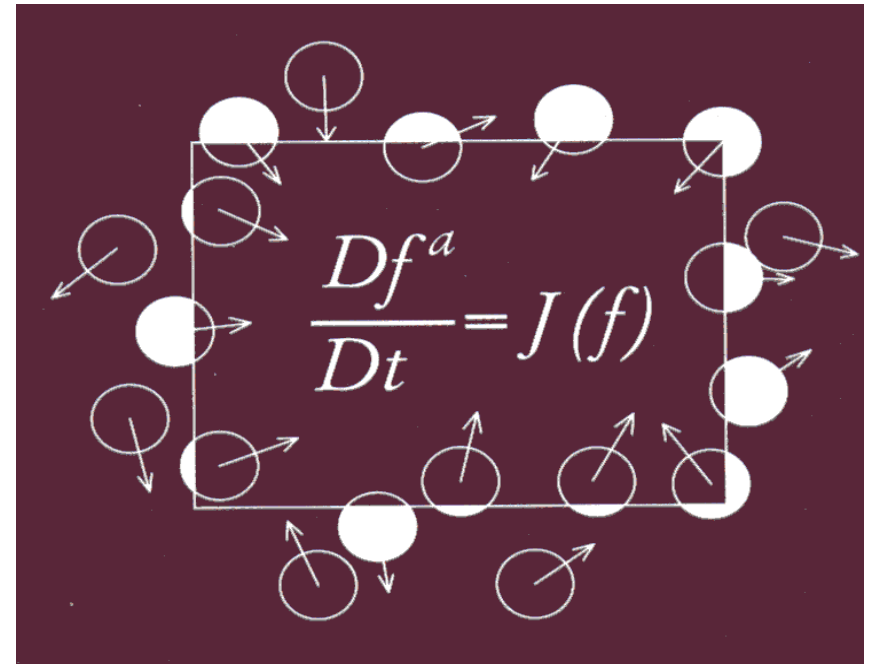

Figure 2. Control loop fluctuations. 
As a result, "Knudsen layers" appear on the borders of the PhIV's. This fact inevitably leads to fluctuations of mass and other hydrodynamic quantities in the PhIV. The existence of such "Knudsen layers" is not related to the choice of a spatial grid and is entirely due to the reduced description of an ensemble of particles of finite diameter. In fact, using the PhIV concept or, what is the same thing, choosing a method for measuring hydrodynamic quantities. This complex of dynamic processes determines the existence of non-local effects in time and space.

What is the order of these effects developing in the border area of the contour? Non-local effects can be demonstrated by animating the movement of particles hard spheres in a neutral gas. Let some volume contains a one-component rarefied gas, in which pair collisions mainly occur. Using a spatial grid, we divide a non-equilibrium physical system into open subsystems-a set of physically infinitesimal volumes. Let's highlight one of these small volumes. To observe the evolution of particles in a selected volume and adjacent small volumes, we will color the particles and trace their movement. Particles in the selected volume are colored blue; there are enough of them to speak about the possibility of a local statistical description at the level of a one-particle distribution function (DF) $f$. In adjacent small volumes, the particles are colored in different colors corresponding to other distribution functions other than $f$. Particles of neighboring volumes are carriers of information about other DFs and, having penetrated into the "blue" volume, adjust to the "blue" DF (if we are talking about relaxation along the translational degrees of freedom) after several collisions. Let, for simplicity, the relaxation process requires only one collision. In other words, a particle that has penetrated into the "blue" volume changes its color to blue after the first collision with the "blue" particle.

You can see firsthand how a borderline "multicolored" area appears in a physically allocated infinitesimal volume ("blue" volume), the characteristic size of which is about the average length of the free motion (Animation 1, https://youtu.be/RvmRW7k-onE).

It is clear that this boundary effect will always exist with the chosen diagnostic method, regardless of the way the system is divided by a set of physically infinitesimal open subsystems.

This fact reflects the existence of non-local effects, proportional to the average path length, the Knudsen number, or the average time between particle collisions.

The structure of the kinetic equation with respect to a single-particle distribution function, generally speaking, looks like this

$$
\frac{D f}{D t}=J^{B}+J^{n l},
$$

where $J^{B}$ is Boltzmann collision integral; $J^{n l}$ is a non-local integral term and $\frac{D}{D t}$ is a the substantial derivative. Since non-local effects are proportional to the 
Knudsen number, it becomes possible to describe non-local effects within the framework of a two-scale approximation. The Boltzmann equation (BE) completely ignores non-local effects and contains only a local collision integral $J^{B}$. But these nonlocal effects are insignificant only in equilibrium systems, where the kinetic approach turns into methods of statistical mechanics.

These nonlocal effects lead to the difficulties of classical Boltzmann physical kinetics.

Even in the "simplest" case, the BE functions on two characteristic scales out of three possible. A natural question arises, what contribution to the $\mathrm{BE}$ is made by the members whose origin is due to this third and smallest scale. It turns out that from the position of the so-called method of many scales even before the uncoupling of the Bogolyubov hierarchy (i.e. before the introduction of any approximations or, if you like, additional physical assumptions) these terms turn out to be of the same order of magnitude as the rest of the BE terms. At the same time, the uncoupling procedure associated with the presence of new members in the $\mathrm{BE}$ leads to the need to choose the direction of time approximation forward in the direction of time or backward. In other words, the question arises whether an approximation should be made based on a deterministic past or a predictable future. By the way, with the "physical" derivation of the BE, such a problem does not arise at all-any direction of approximations leads to the same kind of kinetic equation. These issues are discussed in detail in [1] [2] [3] [4].

In fact, in generalized Boltzmann physical kinetics, a local approximation is proposed for the second collision integral [1] [2] [3] [4].

$$
J^{n l}=\frac{D}{D t}\left(\tau \frac{D f}{D t}\right)
$$

where $\tau$ is proportional to the average time between particle collisions. It is possible to draw an analogy with the Bhatnagar-Gross-Krook approximation (BGK) for $J^{B}$

$$
J^{B}=\left(f^{(0)}-f\right) / \tau,
$$

the popularity of which for the Boltzmann collision integral is explained by the enormous simplification that is achieved when using it. The order of magnitude of the ratio of the second and first terms of the right part (3.1)

$$
J^{n l} / J^{B} \approx O\left(K n^{2}\right)
$$

and with large Knudsen numbers, these terms become one-order. It would seem that for small Knudsen numbers corresponding to the hydrodynamic description, the contribution of the second term of the right part (3.1) can be neglected.

However, this is not the case. During the transition to the hydrodynamic approximation (after multiplying the kinetic equation by collision invariants and subsequent velocity integration), the Boltzmann integral part vanishes, and the second term of the right part (3.1) does not vanish after this integration and gives a one-order contribution under the generalized Navier-Stokes description. 
From a mathematical point of view, neglecting a term containing a small parameter with the highest derivative is not permissible. From a physical point of view, the resulting additional terms proportional to viscosity correspond to Kolmogorov small-scale turbulence. So, it turns out that the integral term turns out to be essential in the theory of transfer processes for both small and large Knudsen numbers.

This fact defines a revolutionary situation in the theory of transfer processes, since the additional terms introduced into the Boltzmann equation cannot be omitted even in the limiting cases of the theory.

Thus, the Boltzmann equation does not even correspond to the class of minimal models of physics, being only a plausible model. Plausible models can lead to acceptable results (often after adjustment with the help of experimental data), but they can also give "failed" results (for example, in the theory of turbulence, the theory of dark matter). Note the interpretation of the generalized BE (GBE) from the standpoint of the theory of fluctuations:

$$
\begin{aligned}
& \frac{D f^{a}}{D t}=J^{B}(f), \\
& f^{a}=f-\tau \frac{D f}{D t} .
\end{aligned}
$$

Equations (3.5), (3.6) have the correct free molecular limit. Thus, there is a fluctuation of the distribution function, and writing in the form (3.5) without taking into account (3.6) makes the BE not closed. From the point of view of the theory of fluctuations, Boltzmann used the simplest closure procedure

$$
f^{a}=f .
$$

So, kinetic theory, taking into account non-local effects, fundamentally leads to fluctuation effects proportional to the average time between collisions. This fact has general theoretical significance and is not related to a specific approximation (3.6). Fluctuation effects occur in any open system bounded by a control surface transparent to particles. Note that the GBE allows you to include in consideration the higher derivatives of DF.

An important methodological issue is the relationship of the generalized BE with classical conservation laws. The macroscopic derivation of conservation laws in continuum mechanics is based on the introduction of a control volume surrounded by an infinitely thin surface. Material points (gas particles) can be located either inside the control volume or outside during their movement, which leads to the classical equations of continuity, motion and energy when recording the balance of mass, momentum and energy flows. In particular, to the continuity equation in the form

$$
\frac{\partial \rho^{a}}{\partial t}+\frac{\partial}{\partial \mathbf{r}} \cdot\left(\rho \mathbf{v}_{0}\right)^{a}=0
$$

where $\rho^{a}$ is the density of the gas $\mathbf{v}_{0}^{a}$-is the hydrodynamic velocity of the flow and $\left(\rho \mathbf{v}_{0}\right)^{a}$ is the density of the impulse flux without taking into account 
fluctuations. Thus, the Boltzmann particles are completely "packed" into the control volume. It would seem that in continuum mechanics it would be possible to abandon the principle of discreteness of matter altogether, considering the medium as continuous in the literal sense of the term. Such an approach is, of course, possible and in hydrodynamics it leads to Euler equations. But if we are going to take into account the effects of viscosity and thermal conductivity, then the situation changes radically. As is known, the dynamic viscosity is proportional to the average time $\tau$ between collisions of particles and a continuous medium in the Eulerian model corresponds to $\tau=0$, which means the absence of viscosity and thermal conductivity. Consequently, the additional terms of the equation describing these effects will be proportional to the average time $\tau$ between collisions of particles, which is reflected by the second term on the left in equation (3.9)

$$
\frac{D f}{D t}-\frac{D}{D t}\left(\tau \frac{D f}{D t}\right)=J^{B}(f) .
$$

And this already leads to very serious consequences in the theory of transfer processes. We know from elementary kinetic theory that the transfer coefficients (for example, the coefficients of viscosity and thermal conductivity) are proportional to $\tau$. This means that the classical equations of hydrodynamics are changing, which acquire additional terms. In fact, the fundamental equations of physics are a consistent system. For example, Maxwell's electrodynamics equations contain current density and charge density. From these equations follows the usual continuity equation for material points. As we can see, Maxwell's equations will contain fluctuation terms of the considered origin. As a result, careful consideration of the effects of advance and delay (or, in other words, non-local effects) leads to a radical transformation of the entire theory of transport processes in physics, including Maxwell's electrodynamics and the theory of turbulent flows.

The Boltzmann equation is undoubtedly a cosmological equation. This was immediately realized by Boltzmann's contemporaries. Indeed, the BE does not contain any restrictions on the "nature" of the system. It can be either the gas contained in the vessel or the universe, as long as these systems fall under the possibility of a single-particle description within the framework of a mechanical model (finite total energy) operating in Euclidean space. As for the possibility of a single-particle description, it did not cause any particular objections from the scientific community. Usually, the mass of a global cosmic formation (such as a Galaxy) is "smeared" to its characteristic size, and in the tradition of Boltzmann kinetic theory (BKT), the average path length of particles and the average time between their collisions are introduced. At the same time, it turns out that the approximation of a single-particle description is acceptable. However, in complete contradiction with the Poincaré-Zermelo theorem, the BKT with the help of the theorem predicts the transition of the system to a state of global thermodynamic equilibrium without the possibility of any fluctuations-in other words, 
to the "thermal death" of the Universe without alternative changes in the process of evolution.

The possibility of the formation of cosmic fluctuations in the mechanical model of the universe follows from the Poincaré-Zermelo theorem, and the evolution of fluctuations can be considered using the generalized Boltzmann kinetic theory, in which local entropy growth is not excluded.

In 1866, Boltzmann published articles in the Sitzungs Berichte Keiserliche Akademie von Wissenschaften (reports of the Vienna Royal Academy of Sciences). In 1872, an article appeared "Weitere Studien über das Wärmegleichgewicht unter Gasmolekulen”, 1872, 66(2), S. 275. The appearance of the Boltzmann equation, which at that time was only 28 years old, is attributed to 1872 . He completed his first job at the age of 21 .

In 1873, at the World Exhibition in Vienna, a memorable conversation took place with Prof. Wroblewski, who recommended Boltzmann to write an accessible course of lectures on kinetic theory. Boltzmann replied that he was unlikely to be able to do it-his eyes were very bad. A magnificent answer followed: "Ein Grund mehr sich zu beeilen" (One more reason to hurry).

The first edition of this book appeared only in 1896 (the preface is dated September 1895). The second edition (or rather, the reissue without changes was published in 1910 after Boltzmann's death). The book appeared with the epigraph: "Alles Vergängliche ist nur ein Gleichniss" (All the past is only a legend. Loosely translated-never regret the past).

Since 1875, L. Boltzmann has been a corresponding member of the Vienna Academy of Sciences, and since 1895-a full member of the Vienna Academy of Sciences.

Boltzmann hanged himself on September 16, 1906 in the town of Duino near Abbacia. Abbacia in those years, as part of the province of Istria, belonged to Austria and was a favorite vacation spot of the Austrian intelligentsia. Abbacia is located on the Adriatic coast at the foot of Mount Maggiore (1396 m.). According to 1890, there were 1192 inhabitants in Abbacia.

In 1852, V. Thomson published the work "On the general tendency to the dissipation of mechanical energy manifested in nature", in which the conclusion about the thermal death of the Universe was formulated for the first time on the basis of a generalized formulation of the first law of thermodynamics. Thomson's conclusions were as follows:

1) In the material world, there is currently a general tendency to waste mechanical energy.

2) The restoration of mechanical energy in its former amount without scattering it in more than an equivalent amount cannot be carried out.

3) In the past, separated by a finite period of time from a certain moment, the Earth was and, after a finite period of time, it will again be in a state unsuitable for human habitation; unless such measures have been carried out in the past and will not be taken in the future, which are impracticable in the presence of laws regulating the known processes taking place now in the material world. 
Clausius (1879), based on Thomson's work, came to the conclusion that the evolution of the universe would lead to "heat death".

These views are refuted by the nonlocal theory of the evolution of matter.

In the early nineties, I worked for a long time in France in Marseille in the Laboratory of Non-equilibrium Media at Aix-en-Provence University (all details in my memoire [7]). The laboratory actively collaborated with the Turbulence Institute (l'Institut de mécanique statistique de la turbulence (IMST)). I made a report [8] on the rigorous theory of turbulence at a seminar of this institute at the invitation of the director of the Institute, Academician Favre (Alexandre Favre). The report was highly appreciated by Favre, who said after the report, "I've been waiting for this report all my life." After one of our subsequent meetings, he somehow recalled the words of A. Poincaré: "Boltzmann was wrong, but his mistake was zero." I replied that Boltzmann was wrong, and his error was not zero.

Academician Mikhail Alexandrovich Leontovich (b. 1903, known for saying that he reads nothing but his works): "I know why Boltzmann hanged himself. He realized that his equation was not correct." A phrase uttered in the Editorial Office of the Physics-Uspekhi.

By 1900, Boltzmann had developed a severe form of asthma; he was experiencing excruciating bouts of illness and suffered greatly from pain. The intense controversy surrounding the molecular kinetic theory affected the state of his nervous system. In 1906, Boltzmann interrupted his lectures and went to the Italian city of Duino for treatment, together with his wife and daughter. All this led to a tragedy: on September 5, 1906, Ludwig Boltzmann hanged himself in a hotel room with a cord from a window curtain while his wife and daughter were on the beach. Boltzmann's suicide is associated with depression caused by the fact that the ideas of statistical physics developed by him at that time were not understood in the physical community. Boltzmann's tombstone has the formula $S=k \ln W$ (engraved with "log" instead of "ln", since the second spelling appeared only 13 years before Boltzmann's death and was not widely used) linking the entropy of the thermodynamic state with the number of corresponding microstates $W$.

\section{Uncertainty Principle, Relativism and Nonlocal Physics}

As it follows from the previous consideration the non local parameter $\tau$ is the mean time of the information transport from a physically infinitely small volume to adjacent physically infinitesimal volume.

Let us consider Animation 2 (https://youtu.be/bqTH0YOMtic) from this point of view. Adjacent physically infinitesimal volumes are denoted in Animation 2 as $\Phi \mathrm{MO}_{1}$ and $\Phi \mathrm{MO}_{2}$. The rigorous approach to derivation of kinetic equation relative to one-particle DF $f\left(K E_{f_{1}}\right)$ is based on employing the hierarchy of Bogolyubov equations [1] [2] [3] [4]. Generalized Boltzmann physical kinetics brings the strict approximation of non-local effects in space and time and after transfer 
to the local approximation leads to parameter $\tau$. In the general case, the parameter $\tau$ is the non-locality parameter; in quantum hydrodynamics, its magnitude is correlated with the "time-energy" uncertainty relation [9]. The uncertainty principle introduced first in 1927 by the German physicist Werner Heisenberg.

The uncertainty principle implies that there is a combination of dynamic characteristic of dynamic systems which cannot be measured with arbitrary certainty, even if all initial conditions are specified.

Let us introduce: reduced Planck constant $\hbar, \Delta x$-the uncertainty in position $x$ of an object (say of a given particle), $\Delta p_{x}$-the uncertainty in momentum $p_{x}$ of an object, $\Delta E$-the uncertainty in energy of an object and $\Delta t$ -the uncertainty in time measurement of an object. In this case it was discovered using the corresponding operators

$$
\begin{aligned}
& \Delta x \cdot \Delta p_{x} \sim \hbar, \\
& \Delta t \cdot \Delta E \sim \hbar .
\end{aligned}
$$

The problem of measurement without restrictions can be solved in the non-dissipative Schrödinger quantum mechanics using the theory of compatible and incompatible observables. For example for the pair " $x, p_{x}$ " the well-known relations exist

$$
\begin{gathered}
\hat{x} \hat{p}_{x} \psi(x)=x\left[-i \hbar \frac{\mathrm{d}}{\mathrm{d} x} \psi(x)\right]=-i \hbar x \frac{\mathrm{d} \psi(x)}{\mathrm{d} x}, \\
\hat{p}_{x} \hat{x} \psi(x)=-i \hbar \frac{\mathrm{d}}{\mathrm{d} x}[x \psi(x)]=-i \hbar\left[\psi(x)+x \frac{\mathrm{d} \psi(x)}{\mathrm{d} x}\right] .
\end{gathered}
$$

We see that in fact we get different results when the operators act in a different order, proving that momentum and position are incompatible observables. Then these dynamic characteristic cannot be measured with the arbitrary accuracy. But the pair " $x, p_{y}$ ” has no corresponding restrictions.

In other words, in the frame of Schrödinger-Madelung [10] [11] [12] quantum mechanics we cannot simultaneously measure both properties within each Equations (4.1), (4.2) to an unlimited level of precision. The more precisely we measure position, the less precisely we are able to simultaneously measure momentum (and vice versa). The more precisely we measure time, the less precisely we are able to simultaneously measure energy (and vice versa).

From the position of nonlocal physics the uncertainty principle is a transparent result which can be explained even on the animation level. Moreover in principle generalized kinetic nonlocal equation (and therefore generalized hydrodynamic equations (GHE)) needn't in using of the "time-energy" uncertainty relation for estimation of the value of the non-locality parameter $\tau$. The "timeenergy" uncertainty relation does not produce the exact relations and from position of non-local physics is only the simplest estimation of the non-local effects.

Really, let us consider:

1) Two neighboring physically infinitely small volumes $\mathrm{PhIV}_{1}$ and $\mathrm{PhIV}_{2}$ in a 
non-equilibrium system.

2) The particles movement in their $1 \mathrm{D}$ motion during invading in the nearest neighboring physically infinitely small volume ( $\mathrm{PhIV}_{1}$ or $\left.\mathrm{PhIV}_{2}\right)$.

The non-locality parameter $\tau$ plays the same role as the transport coefficients in local hydrodynamics. The different models can be introduced for the $\tau$ definition, but the corresponding results not much different like in local kinetic theory for different models of the particles interaction.

Generally speaking parameter $\tau$ is the mean time of the information transfer in adjacent physical volumes.

Obviously the time $\tau$ should tend to diminish with increasing of the velocities $u$ of particles invading in the nearest neighboring physically infinitely small volume $\left(\mathrm{PhIV}_{1}\right.$ or $\left.\mathrm{PhIV}_{2}\right)$ :

$$
\tau=\frac{H_{\tau}}{u^{n}} .
$$

But the value $\tau$ cannot depend on the velocity direction and naturally to tie $\tau$ with the particle kinetic energy, then

$$
\tau=\frac{H_{\tau}}{m u^{2}},
$$

where $H_{\tau}$ is a coefficient of proportionality, which reflects the state of physical system. In the simplest case $H_{\tau}$ is equal to Plank constant $\hbar$ and relation (4.6) became compatible with the Heisenberg relation (4.2).

Look again at the animation 2. We can observe also the particles moving along the surface separating two adjacent physically small volumes. These particles do not introduce the additional information in the nearest volume. This fact explains the existence of compatible observables.

Intermediate conclusion:

The uncertainty principle is the consequence of nonlocal physics on the level of Schrödinger non-dissipative quantum mechanics.

Using these results we are ready to discuss relativistic effects from the position of nonlocal physics. Let us investigate the possible connection between nonlocal physics and special theory of relativity (SRT). The underlying SRT experiment belongs to Albert A. Michelson and Edward W. Morley. The Michelson-Morley experiment was performed and published in November, 1887 (which has tremendous variants in the following years) [13].

The experiment compared the speed of light in perpendicular directions in an attempt to detect the relative motion of matter through stationary luminiferous ether ("ethereal wind"). The result was negative, because Michelson and Morley did not find a significant difference between the speed of light in the direction of movement through the assumed ether and the speed at right angles. This result is generally considered to be the first conclusive evidence against the then-prevalent ether theory, and initiated a line of research that eventually led to a special theory of relativity that excludes stationary ether.

Figure 3 contains the scheme of the Michelson-Morley device. 
Light is sent from the source and propagates with the speed of light $c$ in the aether.

It passes through the half-silvered mirror at the origin at $t=0$. The reflecting mirror is at that moment at distance $L_{\Rightarrow}$ (the length of the interferometer arm) and is moving with velocity $v$.

The beam travel time in the longitudinal direction can be derived as follows:

$$
t_{1}=\frac{L_{\Rightarrow}}{c+v}+\frac{L_{\Rightarrow}}{c-v}=\frac{2 L_{\Rightarrow} c}{c^{2}-v^{2}} .
$$

Calculate the elapsed time for moving in the vertical direction. In one second the aether wind will shift the light on the $v$ meters to the left.

In other words the stationary observer should see the real velocity $u$ of the vertical movement as

$u^{2}+v^{2}=c^{2}, u=\sqrt{c^{2}-v^{2}}$. Then the real rate of convergence of the light with mirror (reflected in Figure 3 as the upper mirror) is $u=\sqrt{c^{2}-v^{2}}$. Moving in the opposite direction leads to the symmetrical situation. Thus,

$$
t_{2}=\frac{2 L_{\Uparrow}}{\sqrt{c^{2}-v^{2}}} .
$$

The time difference is

$$
t_{1}-t_{2}=\frac{2 L_{\Rightarrow} c}{c^{2}-v^{2}}-\frac{2 L_{\Uparrow}}{\sqrt{c^{2}-v^{2}}}=\frac{2 c}{c^{2}-v^{2}}\left[L_{\Rightarrow}-L_{\Uparrow} \frac{\sqrt{c^{2}-v^{2}}}{c}\right] .
$$

or

$$
t_{1}-t_{2}=\frac{2 c}{c^{2}-v^{2}}\left[L_{\Rightarrow}-L_{\Uparrow} \sqrt{1-\frac{v^{2}}{c^{2}}}\right]
$$

\section{Intermediate conclusion:}

The basic relation (4.10) is obtained for the stiff experimental configuration, which does not change its properties and configuration depending on the speed of movement.

H.A. Lorentz was aware in the aether existence. In his lectures on theoretical physics delivered at the University of Leiden he wrote [14], (Chapter 1, Paragraph 8: "Reduction in the direction of movement") he wrote:

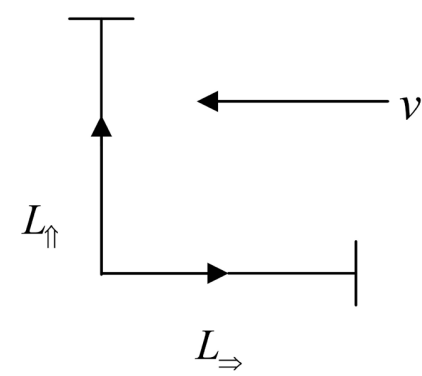

Figure 3. The principal scheme of Michelson-Morley experiment. 
“The negative result of Michelson's experiment can be explained by assuming that the length of the instrument's shoulders changes when rotated to a right angle... This size dependence on the orientation relative to the Earth's motion is not as surprising as it might seem at first. Indeed, the size is determined by molecular forces, and since the latter are transmitted over the aether, on the contrary it would be strange if the state of motion of the latter does not affect the size of bodies. The nature of molecular forces unknown to us; however, if we assume that they are transmitted through the aether in the same way as the electrical forces, then we can build a theory of this reduction, which gives the value of the latter, exactly corresponding to the value necessary to explain the zero effect of the Michelson's experiment. The size of this reduction will be $6.5 \mathrm{~cm}$ for the diameter of the Earth and 1/200 microns for the rod length of $1 \mathrm{~m} . "$

No difficulties to reproduce the Lorentz' estimation; this rod has the length after reduction $L_{r}=L \sqrt{1-\frac{9 \times 10^{2}}{9 \times 10^{10}}}$, m because we have $v_{\text {earth }}=30 \mathrm{~km} / \mathrm{s}$, $c=3 \times 10^{5} \mathrm{~km} / \mathrm{s}$. Then $L_{r}=\sqrt{1-10^{-8}} \mathrm{~m} \approx\left[1-\frac{1}{2} 10^{-8}\right] \mathrm{m}=1 \mathrm{~m}-\frac{1}{200} \mu \mathrm{m}$.

In other words relation (4.10) obtained for the closed thermodynamic system without taking into account the direct influence of physical vacuum PV (or in old terminology) luminiferous aether. In principal we consider the open thermodynamic system interacting with physical vacuum (PV). But the relation (4.7), (4.8) obtained for classic dynamic system without taking into account the direct influence of PV on the mentioned system.

Therefore we have only two possibilities:

1) We need take into account the real processes of "matter-physical vacuum" interaction.

2) Nevertheless we intend to construct simplified theory excluding the direct PV influence. This fact leads to kinematic relation

$$
L_{\Rightarrow}=L_{\Uparrow} \sqrt{1-\frac{v^{2}}{c^{2}}} .
$$

if $t_{1}-t_{2}=0$.

Let us consider this situation in detail from the position of the nonlocal physics. The Michelson installation can be considered as a device delivering particles moving with the velocities $v$ in the open probe contour filled by PV. The corresponding nonlocal parameter is (see also (4.6))

$$
\tau_{D}=\frac{H_{D}}{v^{2}} .
$$

Now we can rewrite the relation (4.10) in the form

$$
t_{1}-t_{2}=\frac{2 c}{c^{2}-v^{2}}\left[L_{\Rightarrow}-L_{\Uparrow} \sqrt{1-\frac{v^{2}}{c^{2}}}\right]=\frac{2 c \tau_{D}}{\tau_{D} c^{2}-H_{D}}\left[L_{\Rightarrow}-L_{\Uparrow} \sqrt{1-\frac{H_{D}}{\tau_{D} c^{2}}}\right] .
$$

Michelson's experiment assumes that the length of the instrument's shoulders does not change when rotated to a right angle. 
In this case from the position of nonlocal physics

$$
t_{1}-t_{2}=0
$$

only if

$$
H_{D} \rightarrow 0, L_{\Rightarrow}=L_{\Uparrow}
$$

or if the PV viscosity tends to zero.

Conclusion:

1) Special relativistic theory contradicts the Heisenberg's uncertainty principle.

2) The uncertainty principle reflects the existence of Physical Vacuum.

3) Special theory of relativity is kinematic theory which allows avoiding PV effects in the explicit form from consideration. Lorentz was right.

As we can see, the Lorentz-Poincare special theory of relativity is a mathematical apparatus that allows avoiding direct consideration of the effects of physical vacuum in the calculations of the evolution of physical systems. This brilliant idea can also be implemented taking into account non-local effects [3].

Criticism of general relativity (GR) is well known topic in theoretical physics (see for example [15]). No reason to discuss here the shortcomings of GRT.

\section{Final Remarks and Conclusions}

The following results cannot be obtained on the elementary level of consideration. The corresponding strict results can be found in monographs [1] [2] [3] [4]. Nevertheless here I formulate some significant results without additional explanations. What solutions do the equations of Unified Theory (UT, Unified Theory of Transport Processes) lead to?

Let's formulate conclusions from the solutions at a qualitative level:

1) Nonlocal physics returns $96 \%$ of matter and energy to humanity. Therefore, classical local physics of dissipative processes is a deep special case of nonlocal physics. Let us note the fundamentally important stages in the development of nonlocal physics.

In 1926, the brilliant article by Madelung, which I have repeatedly quoted, appeared which turned Schrödinger's quantum postulate into hydrodynamics. In other words, the evolution of a single bound electron turned out to be possible to interpret as some effective flow.

In 1964, John Stuart Bell [16] established that local statistical theories of dissipative processes are incorrect in principle.

In 2007, B.V. Alexeev established [17] [18] that the Schrödinger equation and its hydrodynamic Madelung form are a consequence of nonlocal kinetic equations as a deep special case of nonlocal equations. This meant that the generalized physical kinetics I had created earlier was extended to quantum physics in the form of quantum hydrodynamics.

2) Nonlocal quantum hydrodynamics leads to non-collapsing solitons and, as a consequence, to the theory of high-temperature superconductivity [19] [20]. 
3) The developed nonlocal unified statistical theory of dissipative structures has a hydrodynamic shape defined by the genesis of Generalized Hydrodynamic Equations (GHE). GHE are extremely important for astrophysics special cases when density $\rho \rightarrow 0$ (the initial stage of evolution of the Universe, the Big Bang; transport processes in physical vacuum) and when density $\rho \rightarrow \infty$ (evolution of the black hole). Both limiting cases have no physical or mathematical meaning in "classical" hydrodynamics including General Relativity

4) The birth of the Universe is accompanied by an explosion of the primary vacuum (PV) - a medium that does not contain matter, electromagnetic and gravitational fields. A small disturbance is enough for an explosion. From a mathematical point of view, there is Hadamard instability. At the same time, there is a well-known effect in mathematics-the smaller the disturbance, the greater the efficiency of the explosion.

5) The primary vacuum is the initial state of the Physical Vacuum (PV). Until now, we could claim that Physical Space (PS) consists of matter and a field. Now we are talking about the existence of the trinity: Matter, Field and Physical Vacuum. All components of the PS can experience mutual transformations.

6) The set of dynamic characteristics of the initial stage of the evolution of the PV contains the speed, the energy of the unit volume of the PV and the force acting on the unit volume of the PV.

7) The explosion "works" in the anti-gravity mode, since there is no substance yet. In the terminology currently in use, PV energy is dark energy.

8) After the birth of matter and gravity, the Hubble expansion mode appears. From the point of view of solving the UT equations, the Hubble expansion is the gravitational self-capture of matter under the conditions of a small influence of central gravitational masses. The Hubble phenomenon is characteristic not only as a dynamic effect characteristic of the entire Universe, but also for individual galactic systems-"Hubble boxes", in particular, for the Local Group-the system of galaxies to which our Milky Way belongs.

9) The classical Hubble expansion is characterized by a proportional increase in the expansion velocity of the group depending on the distance from the main center of gravity. The proportionality coefficient is the Hubble constant $\left(\mathrm{H}_{0}\right)$. Naturally, this means that the expansion occurs with acceleration even at a constant coefficient $\mathrm{H}_{0}$. However, recent observations have established (S. Perlmutter, A. Riess (USA) and B. Schmidt (Australia); Nobel Prize 2011) that the expansion of the Universe at this stage of evolution is accompanied by an increase $\mathrm{H}_{0}$.

10) Is this mode unique? There are reasons to give a negative answer. Astronomical observations of the evolution of a Local Group of galaxies can be interpreted as the scattering of Dwarf Galaxies in the mode of the coefficient $\mathrm{H}_{0}$ increasing with distance.

11) There is a feeling that the role of the Creator is reduced to the introduction of the initial disturbance into the PV. 
12) But is it possible to avoid the concept of initial perturbation in principle? Generally speaking, it is possible. Solving the UT equations in the Hubble expansion mode allows you to calculate the Hubble coefficient at the same time. It turns out that there really are modes when the Hubble coefficient increases. But there are modes when it decreases and even becomes negative. The latter means that the universe is beginning to "collapse".

13) The regime of the pulsating Universe no longer requires additional intervention, if we recognize that the primary vacuum has always existed, and explosions are caused by random fluctuations of the PV, which are inherent in any dynamical system. It is possible that there are local volumes of PV, the dynamics of which resembles the dynamics of Hubble boxes.

14) The life of our universe is conditioned by the existence of world constants, among which are the fine structure constant, Planck constant, Boltzmann constant. Other constants represent a completely different evolution of the universe in a specific dynamic scenario of the pulsation cycle. The discovery of voids means that other explosions of the physical vacuum are possible, leading to other world constants and parallel universes.

15) The birth of our universe with a specific set of world constants is the product of a specific fluctuation in the cycle. From this point of view, we can say that we were lucky that we got into the "right fluctuation".

16) The orbital motion of astronomical objects according to the Vera Rubin model (and not according to Kepler) is a direct consequence (an exact analytical result) of non-local physics. I once again suggest that NASA measure the orbital velocity of motion inside one of Saturn's rings. The internal orbital velocity of the particles in the ring must be constant (that is, in accordance with Vera Rubin's model).

\section{Conflicts of Interest}

The author declares no conflicts of interest regarding the publication of this paper.

\section{References}

[1] Alexeev, B.V. (2004) Generalized Boltzmann Physical Kinetics. Elsevier Science, Amsterdam, 368 p. https://doi.org/10.1016/B978-0-444-51582-7.X5025-5

[2] Alexeev, B.V. (2015) Unified Non-Local Theory of Transport Processes. Elsevier, Amsterdam, 644 p. https://doi.org/10.1016/C2014-0-00360-7

[3] Alexeev, B.V. (2016) Unified Non-Local Relativistic Theory of Transport Processes. Elsevier, Amsterdam, 455 p. https://doi.org/10.1016/C2016-0-00437-0

[4] Alexeev, B.V. (2017) Nonlocal Astrophysics: Dark Matter, Dark Energy, Physical Vacuum. Elsevier, Amsterdam, 454 p. https://doi.org/10.1016/C2016-0-05329-9

[5] Alexeev, B.V. (2021) Nonlocal Physics in the Wave Function Terminology. Journal of Applied Mathematics and Physics, 9, 2889-2908.

https://doi.org/10.4236/jamp.2021.911183

[6] Ренан, Э. (1907) Антихрист. С.-Петербург, Издательство Пирожкова. М.В. (In 
Russian, Translation from the Second French Edition). Pirozhkof, St. Petersburg.

[7] Alexeev, B. (2017) What Is It-Happiness? Touches to the Portrait of the Outgoing Original (Russian Edition) PRODUCT Details. LAP LAMBERT Academic Publishing, Saarbrücken.

[8] Alexeev, B.V. (1994) The Generalized Boltzmann Equation, Generalized Hydrodynamic Equations and Their Applications. Philosophical Transactions of the Royal Society of London. Series A: Physical and Engineering Sciences, 349, 417-443. https://doi.org/10.1098/rsta.1994.0140

[9] Heisenberg, W. (1927) Über den anschaulichen Inhalt der quantentheoretischen Kinematik und Mechanik. Zeitschrift für Physik, 43, 172-198.

https://doi.org/10.1007/BF01397280

[10] Schrödinger, E. (1926) Quantisierung als eigenwertproblem. Annalen der Physik, 384, 361-376. https://doi.org/10.1002/andp.19263840404

[11] Madelung, E. (1926) Eine anschauliche Deutung der Gleichung von Schrödinger. Naturwissenschaften, 14, 1004. https://doi.org/10.1007/BF01504657

[12] Madelung, E. (1927) Quantentheorie in Hydrodynamischer Form. Zeitschrift für Physik, 40, 322-326. https://doi.org/10.1007/BF01400372

[13] Michelson, A.A. and Morley, E.W. (1887) On the Relative Motion of the Earth and the Luminiferous Ether. American Journal of Science, s3-34, 333-345.

https://doi.org/10.2475/ajs.s3-34.203.333

[14] Lorentz, H.A., Bremekamp, H., Bruins, E.D., Reudler, J., Crommelin, C.A., Fokker, A.D., et al. (1901-1902) Aether Theories and Aether Models. Lectures on Theoretical Physics Delivered at the University of Leyden, Vol. 1, Macmillan and Co., Ltd., London.

[15] Logunov, A.A., Loskutov, Yu. M. and Mestvirishvili, M.A. (1987) Relativistic Theory of Gravitation and Criticism of General Relativity. Theoretical and Mathematical Physics, 73, 1131-1148. https://doi.org/10.1007/BF01017582

[16] Bell, J.S. (1964) On the Einstein Podolsky Rosen Paradox. Physics Physique Fizika, 1, 195-200. https://doi.org/10.1103/PhysicsPhysiqueFizika.1.195

[17] Alexeev, B.V. (2008) Generalized Quantum Hydrodynamics and Principles of Non-Local Physics. Journal of Nanoelectronics and Optoelectronics, 3, 143-158. https://doi.org/10.1166/jno.2008.207

[18] Alexeev, B.V. (2008) Application of Generalized Quantum Hydrodynamics in the Theory of Quantum Soliton's Evolution. Journal of Nanoelectronics and Optoelectronics, 3, 316-328. https://doi.org/10.1166/jno.2008.311

[19] Alexeev, B.V. (2021) Nonlocal Theory of High-Temperature Superconductivity. Journal of Modern Physics, 12, 552-593. https://doi.org/10.4236/jmp.2021.125037

[20] Alexeev, B.V. (2021) Nonlocal Theory of Superconductivity (Monograph). Lambert, Saarbrücken. 